\title{
BMJ Soft drink intake and progression Open of radiographic knee osteoarthritis: data from the osteoarthritis initiative
}

\author{
Bing Lu, ${ }^{1}$ Oneeb Ahmad, ${ }^{2}$ Fang-Fang Zhang, ${ }^{3}$ Jeffrey B Driban, ${ }^{4}$ Jeffrey Duryea, ${ }^{1}$ \\ Kate L Lapane, ${ }^{5}$ Timothy McAlindon, ${ }^{4}$ Charles B Eaton ${ }^{2,6}$
}

To cite: Lu B, Ahmad 0 , Zhang F-F, et al. Soft drink intake and progression of radiographic knee osteoarthritis:

data from the osteoarthritis initiative. BMJ Open 2013;3: e002993. doi:10.1136/ bmjopen-2013-002993

- Prepublication history for this paper is available online. To view these files please visit the journal online (http://dx.doi.org/10.1136/ bmjopen-2013-002993)

Received 3 April 2013 Revised 11 June 2013 Accepted 14 June 2013

\footnotetext{
${ }^{1}$ Brigham \& Women's Hospital and Harvard Medical School, Boston, Massachusetts, USA ${ }^{2}$ Center for Primary Care and Prevention, Memorial Hospital of Rhode Island, Pawtucket, Rhode Island, USA

${ }^{3}$ Friedman School of Nutrition Science and Policy, Tuft University, Boston, Massachusetts, USA

${ }^{4}$ Tufts Medical Center, Boston, Massachusetts, USA ${ }^{5}$ University of Massachusetts Medical School, Worcester, Massachusetts, USA ${ }^{6}$ Departments of Family Medicine and Epidemiology, The Warren Alpert Medical School of Brown University, Providence, Rhode Island, USA
}

Correspondence to Dr Bing Lu; blu1@partners.org

\begin{abstract}
Objectives: We examine the prospective association of soft drink consumption with radiographic progression of knee osteoarthritis (OA).

Design: Prospective cohort study.

Setting: This study used data from the osteoarthritis initiative (OAI).

Participants: In OAI, 2149 participants with radiographic knee $0 \mathrm{~A}$ and having dietary data at baseline were followed up to 12, 24, 36 and 48 months.

Measures: The soft drink consumption was assessed with a Block Brief Food Frequency Questionnaire completed at baseline. To evaluate knee $\mathrm{OA}$ progression, we used quantitative medial tibiofemoral joint space width (JSW) based on plain radiographs. The multivariate linear models for repeated measures were used to test the independent association between soft drink intake and the change in JSW over time, while adjusting for body mass index and other potential confounding factors.
\end{abstract}

Results: In stratified analyses by gender, we observed a significant dose-response relationship between baseline soft drink intake and adjusted mean change of JSW in men. With increasing levels of soft drink intake (none, $\leq 1,2-4$ and $\geq 5$ times/week), the mean decreases of JSW were $0.31,0.39,0.34$ and $0.60 \mathrm{~mm}$, respectively. When we further stratified by obesity, a stronger dose-response relationship was found in nonobese men. In obese men, only the highest soft drink level ( $\geq 5$ times/week) was associated with increased change in JSW compared with no use. In women, no significant association was observed.

Conclusions: Our results suggest that frequent consumption of soft drinks may be associated with increased OA progression in men. Replication of these novel findings in other studies demonstrating the reduction in soft drink consumption leads to delay in $O A$ progression is needed.

\section{INTRODUCTION}

Osteoarthritis (OA), a slowly progressing disease characterised by pain, deformity and loss of function, is the major cause of physical

\section{ARTICLE SUMMARY}

Article focus

- To examine the prospective association of soft drink consumption with radiographic progression of knee osteoarthritis $(\mathrm{OA})$.

- To examine gender differences in effect of soft drink on $\mathrm{OA}$ progression.

Key messages

- There is a significant dose-response relationship between soft drink intake and radiographic knee OA progression in men, but not in women.

- A stronger relationship was found in non-obese men.

Strengths and limitations of this study

- The prospective design, large number of patients with knee $O A$ and the quantitative measures of structural change from sophisticated image processing technology.

- Consistent findings using semiquantitative as well as quantitative measures of OA progression.

- Residual confounding may exist for this observational study.

Soft drink consumption was based on self-report.

disability in older people. ${ }^{1}$ Nearly 27 million have clinical OA in the USA. ${ }^{2}$ With the aging of the population, the healthcare burden from $\mathrm{OA}$ is expected to increase dramatically during the next few decades. ${ }^{3}$ However, little is known about the course of OA progression over time in patients with OA. It is, therefore, of great importance to identify modifiable risk factors for OA progression. Over the past few decades, many observational studies have examined risk factors for the incidence and progression of knee OA. Several risk factors (ie, obesity, joint injury and certain sports) have been found to be strongly associated with an increased risk for incident knee OA. ${ }^{45}$ However, findings on risk factors for OA progression have been inconclusive. Except for the level of serum hyaluronic acid and generalised OA, no other risk factor has 
been consistently associated with the risk of OA progression. ${ }^{6} 7$

Soft drink consumption has increased rapidly across the globe in recent decades. ${ }^{8}$ Sugar sweetened beverages intake is a significant contributor to weight gain and has been associated with increased risk of obesity, type 2 diabetes, cardiovascular disease and poor bone health. ${ }^{9-11}$ Soft drinks may displace essential nutrients and contribute to overall poorer diets, ${ }^{12}$ while low consumption of vitamin $\mathrm{D}$ and antioxidant micronutrients may increase the risk for progression of knee OA. ${ }^{13}{ }^{14}$ To our knowledge, no study has linked soft drink consumption to OA progression. We examined the prospective association between consumption of soft drinks and progression of knee OA using data from the osteoarthritis initiative (OAI).

\section{METHODS}

\section{Subjects}

OAI was launched in 2002 by the National Institute of Health to develop resources for the identification of new biomarkers and treatment targets for knee OA. The objective of the OAI was to pool public and private scientific expertise and funding to collect, analyse and make widely available the largest research resource to date of clinical data, radiological information and biospecimens from those at risk for or with knee OA. The OAI began enrolling people aged 45 through 79 years in 2004 and followed them annually for the development or progression of OA. The clinical sites involved were located in Baltimore, Maryland; Columbus, Ohio; Pittsburgh, Pennsylvania and Pawtucket, Rhode Island. OAI has been a longitudinal study of 4796 participants with either established knee OA or significant risk factors for the development of knee OA followed over an 8-year period. ${ }^{15}$ The follow-up rate was $>90 \%$ over the first 48 months. The detailed OAI protocol can be found elsewhere. ${ }^{16}$

For the current study, we included individuals with medial radiographic knee $\mathrm{OA}$ in at least one knee at baseline. We excluded knees with severe radiographic OA defined as the baseline Kellgren and Lawrence (KL) grade of 4 or those with primarily lateral joint space narrowing (JSN), and knees in which the difference of rim distance (from tibial plateau to tibial rim closest to femoral condyle) between follow-up and baseline visits were $\geq 2 \mathrm{~mm}$ to minimise possible measurement error of radiographic data. The 2149 participants (3066 knees) with KL grade of 2 or 3 and having dietary data at baseline constituted the study sample. Follow-up at 12, 24, 36 and 48 months were included in this analysis. The overall loss to follow-up rate was $16.8 \%$ over the study period.

\section{Radiographic progression of $\mathrm{OA}$}

In OAI, current radiographic assessment techniques on plain radiographs involved semiquantitative as well as quantitative assessment of JSN. For the semiquantitative approach, the Osteoarthritis Research Society International grade (OARSI grade: $0=$ no JSN, $1=$ definite JSN to $3=$ severe JSN) has been widely used to measure progression of $\mathrm{OA} .{ }^{17}$ For these analyses, we used the publicly available semiquantitative JSN readings (kXR_SQ_BU, V.11/07/2011, http:/ /oai.epi-ucsf.org). Recently, a quantitative approach has been used to provide a precise measure of joint space width (JSW) in millimetres between the adjacent bones of the knee. ${ }^{18}{ }^{19}$ Multiple JSWs were measured at fixed locations along the joint in medial compartment, denoted as JSW(x), at 0.025 intervals for $\mathrm{x}=0.15-0.30$. The reproducibility of this technique and the responsiveness to change have been documented elsewhere, ${ }^{18} 20$ including one study using OAI data which demonstrated a responsiveness that compared favourably with MRI. ${ }^{20}$ We used medial JSW at $\mathrm{x}=0.25$ with the best responsiveness of change to quantify the progression of $\mathrm{OA} .{ }^{20}$ We define the repeated measures of the decreases of JSW from baseline to 12, 24, 36 and 48 months as the outcome variable.

\section{Assessment of soft drink consumption}

Usual dietary intakes of foods and nutrients including soft drink consumption were assessed at baseline with a Block Brief Food Frequency Questionnaire (FFQ) including 60 food items in OAI. ${ }^{21}$ The participants were asked how often they had consumed regular soft drinks/ bottled drinks (not diet drinks) in the past 12 months (coded as: never, a few times/year, once/month, 2-3 times/month, once/week, twice/week, 3-4 times/week, 5-6 times/week and every day; variable name: V00FFQ69, http://oai.epi-ucsf.org). Based on previous studies $^{\widehat{9-11}}$ we grouped these into four categories: none, $\leq 1,2-4$ and $\geq 5$ times/week. Similar questionnaires were used to collect the frequencies of other beverages intake, such as, milk, juice, tea and coffee, etc. This brief FFQ has been validated against three 4-day records in a group of middle-aged women, and against two 7-day records in a group of older men. The absolute value of macronutrients estimated by the reduced questionnaire was a slightly lower than food-record estimates, but most micronutrients were not underestimated. ${ }^{21} 22$

\section{Information on covariates}

Baseline demographic and socioeconomic factors include race/ethnicity, age, sex, marital status, education level, employment status, annual income and social support. Individuals were classified as African-American, white or other racial/ethnic group based on self-report. Education level was categorised as high school or less, college and above college. General clinical parameters include current smoking, alcohol consumption, history of traumatic knee injury and knee surgery, self-reported gout, body mass index (BMI), physical activity, weight change, milk and juice intake, total energy intake and baseline disease severity (KL grade). Physical activity was assessed by using the Physical Activity Scale for the Elderly (PASE), an established questionnaire for 
measuring physical activity in older individuals that has also been validated in younger participants. ${ }^{23}{ }^{24}$ Alcohol consumption (pure alcohol in $\mathrm{g} /$ day) was assessed at baseline including separate items for beer, wine and liquor in OAI. In addition, we also adjusted for changes of the beam angles and rim distances (from tibial plateau to tibial rim closest to femoral condyle between follow-up visits and baseline) which indicate kneepositioning consistency for X-ray examination.

\section{Statistical analysis}

First we performed exploratory analyses of all variables of interest including the exposure (frequency of soft drink intake), radiographic structural measures (KL grade, JSN score and JSW) and potential confounders described above. Descriptive statistics such as the minimum, maximum, median and mean for each continuous variable and frequency table for each categorical variable was used to summarise the data as well as detect outliers, data entry mistakes and missing values.

The primary analysis was to assess the influence of soft drink consumption on the change in JSW over the study period. The primary outcomes were repeated measures of the JSW decreases from baseline to 12, 24, 36 and 48 months, respectively. The initial analyses were unadjusted comparisons of the changes of JSW over time among levels of soft drink intake using analysis of variance and multivariate analysis of variance. Then separate multivariate models for repeated measures by men and women were used to test the independent association between soft drink intake and the decrease in JSW over time, while adjusting for BMI, physical activity, baseline disease severity and potential confounding factors described above. Owing to the hierarchical structure of the data (each participant has two knees over multiple time points), we used general linear mixed models to account for within participant correlation. The final covariance models were evaluated using Akaike's information criterion and Bayesian information criterion. BMI has been an important factor related to soft drink intake as well as OA progression. ${ }^{9}{ }^{25}$ To examine the possible effect modifications, we further performed stratified analyses by obesity $\left(\mathrm{BMI} \geq 30 \mathrm{~kg} / \mathrm{m}^{2}\right)$ and also adjusted BMI within each category to reduce the possible residual confounding bias. In addition, the association of soft drink consumption with JSW change may also be mediated through BMI. The indirect effect of $\mathrm{BMI}$ was evaluated using Sobel test ${ }^{26}$

In addition, we also used the first increase of the OARSI JSN grade as the endpoint. We developed a Cox proportional hazards model to assess independent association between soft drink intake and the JSN score change after controlling for other covariates. For each participant, the time of follow-up was calculated from the baseline date to the date of the first increase of JSN grade, death or end of the study, whichever came first. The discrete likelihood method was used for ties of the failure times in the models. We used a robust sandwich covariance estimate to account for the intraclass dependence within individual patients. ${ }^{27}$ Participants who indicated no soft drink consumption in the past year were chosen as the reference group for all analyses. Adjusted HRs with 95\% CIs were used to evaluate the strength of the associations. The proportional hazard assumption was tested based on the smoothed plots of the scaled Schoenfeld residuals. Data analyses were performed using SAS V.9.2 (SAS Institute, Cary, North Carolina, USA).

\section{RESULTS}

In this study, we studied 2149 participants from OAI having a total of 3066 eligible knees. All categories of soft drink intake were represented in participants at baseline (none, $\mathrm{n}=687$; $\leq 1$ times/week, $\mathrm{n}=976$; 2-4 times/week, $\mathrm{n}=285 ; \geq 5$ times/week, $\mathrm{n}=201$ ). Baseline characteristics of participants are shown in table 1 according to levels of soft drink intake. Compared with no soft drink use, high soft drink users were more likely to be men, between 45 and 54 years, not married, not employed, current smokers and have lower education and household income and higher BMI.

Results of multivariable analyses are shown in table 2 in men and women. We observed a significant doseresponse relationship in men between soft drink intake and adjusted mean decreases of JSW in men ( $p$ trend $<0.001$ ) after controlling for age, race, education, marital status, household income, employment, BMI, physical activity, follow-up time, knee injury and knee surgery, smoking, milk and juice intake, total energy intake, baseline KL grade, weight change, the changes of rim distance and beam angle. With increasing levels of soft drink intake (none, $\leq 1,2-4$, and $\geq 5$ times/ week), the mean decreases of JSW were $0.31,0.39,0.34$ and $0.60 \mathrm{~mm}$, respectively. When we stratified by obesity, a stronger dose-response relationship was found (decreases of JSW were $0.24,0.38,0.32,0.62$, respectively) in non-obese men. In obese men, only the highest soft drink level ( $\geq 5$ times/week) was associated with increased JSW change compared with no use. The effect of soft drink consumption was different between men and women ( $\mathrm{p}$ for interaction $=0.003$ ). No significant association was observed in women. Table 3 shows the multivariable adjusted HR of OA progression evaluated by time to the first increase of semiquantitative OARSI score. Consistent with JSW analyses, we found that the increasing soft drink intake was associated with the increasing rate of OA progression in men but not in women. Compared with no soft drink intake, the HR for $\leq 1,2-4$ and $\geq 5$ times/week were 1.56 (95\% CI 1.13 to 2.16), 1.55 (95\% CI 1.02 to 2.35 ) and 2.05 (95\% CI 1.32 to 3.19 ), respectively, in men ( $p$ trend=0.002). When we stratified by obesity, a dose-response relationship was observed in non-obese as well as obese men. The HRs were 2.30 (95\% CI 1.22 to 4.34 ) and 1.90 (95\% CI 1.03 to 3.52 ), respectively, with the soft drink intake $\geq 5$ 
Table 1 Baseline characteristics of participants with radiographic knee osteoarthritis according to levels of soft drink intake

\begin{tabular}{|c|c|c|c|c|c|c|}
\hline \multirow[b]{2}{*}{ Variables } & \multirow[b]{2}{*}{ Total $\mathbf{N}=\mathbf{2 1 4 9}$} & \multicolumn{5}{|c|}{ Soft drink intake (times/week) } \\
\hline & & None, $n=687$ & $\leq 1, \mathrm{n}=976$ & $2-4, n=285$ & $\geq 5, n=201$ & p Value \\
\hline Age (years), mean (SD) & $62.4(9.0)$ & $63.9(8.9)$ & $62.7(8.8)$ & $61.6(9.1)$ & $58.0(8.0)$ & $<0.001$ \\
\hline \multicolumn{7}{|l|}{ Race/ethnicity (\%) } \\
\hline Non-hispanic White & 76.04 & 83.70 & 77.36 & 62.46 & 62.69 & \\
\hline Non-hispanic Black & 20.75 & 12.95 & 19.36 & 34.74 & 34.33 & \\
\hline$\leq$ High school & 18.44 & 14.12 & 17.21 & 27.72 & 26.00 & \\
\hline College & 45.90 & 46.00 & 46.31 & 44.56 & 45.50 & \\
\hline Above college & 35.66 & 39.88 & 36.48 & 27.72 & 28.50 & $<0.001$ \\
\hline \multicolumn{7}{|l|}{ Household income (\%) } \\
\hline$<25 \mathrm{k}$ & 15.17 & 11.51 & 15.04 & 18.42 & 23.56 & \\
\hline Employed (\%) & 58.21 & 53.31 & 57.89 & 58.60 & 69.15 & 0.002 \\
\hline \multicolumn{7}{|l|}{ Smoke (\%) } \\
\hline Never & 54.07 & 51.53 & 53.79 & 59.65 & 56.22 & \\
\hline Current & 6.38 & 4.51 & 5.43 & 7.02 & 16.42 & \\
\hline Past & 39.55 & 43.96 & 40.78 & 33.33 & 27.36 & $<0.001$ \\
\hline \multicolumn{7}{|l|}{ Alcohol (g/day) } \\
\hline $0-<5$ & 66.08 & 63.75 & 66.19 & 68.77 & 69.65 & \\
\hline $5-<10$ & 10.80 & 11.21 & 10.45 & 12.98 & 7.96 & \\
\hline $10+$ & 23.12 & 25.04 & 23.36 & 18.25 & 22.39 & 0.056 \\
\hline PASE, mean (SD) & $157(82)$ & $147(77)$ & $161(82)$ & $165(90)$ & $163(83)$ & 0.001 \\
\hline \multicolumn{7}{|l|}{ BMI $\left(\mathrm{kg} / \mathrm{m}^{2}\right)(\%)$} \\
\hline \multicolumn{7}{|l|}{$\mathrm{KL}$ grade (index knee) (\%) } \\
\hline None & 17.19 & 22.27 & 12.62 & 17.31 & 21.89 & \\
\hline$\leq 1$ & 30.52 & 28.09 & 32.41 & 29.33 & 31.34 & \\
\hline $2-4$ & 21.39 & 20.52 & 22.87 & 20.49 & 18.41 & \\
\hline$\geq 5$ & 30.89 & 29.11 & 32.10 & 32.86 & 28.36 & 0.657 \\
\hline
\end{tabular}

times/week compared with no intake. However, no significant association was found in women. In addition, our mediation analysis indicated a modest $(3.2 \%)$ indirect effect through BMI ( $p$ for Sobel test=0.098). In sensitivity analyses, additional adjustment for alcohol consumption and a history of gout in the above models did not change the results.

\section{DISCUSSION}

In this 48-month follow-up study of people with radiographic knee OA, we found a positive association with a significant dose-response relationship between soft drink consumption and structural progression of knee OA measured by semiquantitative as well as quantitative JSN independent of $\mathrm{BMI}$ and other potential risk factors in men, but not in women.

Knee OA progression has been thought to involve multiple mechanisms besides cartilage loss including changes in bone composition, shape, as well as proprioreception, ${ }^{28} 29$ which might be subject to the influences of macro and micronutrients in the diet. McAlindon et $a l^{14}$ reported that a higher consumption of antioxidant micronutrients, especially vitamin $\mathrm{C}$, might decrease cartilage loss and $\mathrm{OA}$ progression, and low vitamin $\mathrm{D}$ intake and low serum vitamin $\mathrm{D}$ levels might increase the risk for progression of knee OA. ${ }^{13}$ However, no study investigated the association of soft drink consumption and progression of OA. Sugar sweetened beverages intake is a significant contributor to weight gain 
Table 2 Adjusted mean (SE) decreases of joint space width (JSW) by soft drink intake*

\begin{tabular}{|c|c|c|c|c|c|c|c|c|}
\hline \multirow[b]{2}{*}{ Soft drinks (times/week) } & \multicolumn{4}{|l|}{ Men } & \multicolumn{4}{|c|}{ Women } \\
\hline & $\mathbf{N}$ & $\Delta \mathrm{JSW}(\mathrm{mm})^{\star}$ & p Value & p trend & $\mathbf{N}$ & $\Delta \mathrm{JSW}(\mathrm{mm})^{\star}$ & p Value & $p$ trend \\
\hline \multicolumn{9}{|l|}{ Overall } \\
\hline None & 202 & $0.31(0.03)$ & Referent & & 485 & $0.32(0.02)$ & Referent & \\
\hline$\leq 1$ & 439 & $0.39(0.02)$ & 0.055 & & 537 & $0.33(0.02)$ & 0.626 & \\
\hline $2-4$ & 140 & $0.34(0.04)$ & 0.540 & & 145 & $0.36(0.04)$ & 0.331 & \\
\hline$\geq 5$ & 107 & $0.60(0.05)$ & $<0.001$ & $<0.001$ & 94 & $0.30(0.05)$ & 0.751 & 0.756 \\
\hline \multicolumn{9}{|l|}{ Non-obese } \\
\hline None & 113 & $0.24(0.04)$ & Referent & & 279 & $0.30(0.03)$ & Referent & \\
\hline$\leq 1$ & 266 & $0.38(0.03)$ & 0.010 & & 277 & $0.30(0.03)$ & 0.946 & \\
\hline $2-4$ & 85 & $0.32(0.05)$ & 0.236 & & 60 & $0.35(0.06)$ & 0.395 & \\
\hline$\geq 5$ & 53 & $0.62(0.07)$ & $<0.001$ & $<0.001$ & 32 & $0.30(0.07)$ & 0.955 & 0.681 \\
\hline \multicolumn{9}{|l|}{ Obese } \\
\hline None & 89 & $0.40(0.05)$ & Referent & & 206 & $0.34(0.03)$ & Referent & \\
\hline$\leq 1$ & 173 & $0.41(0.03)$ & 0.993 & & 260 & $0.37(0.03)$ & 0.471 & \\
\hline $2-4$ & 55 & $0.38(0.06)$ & 0.749 & & 85 & $0.38(0.05)$ & 0.518 & \\
\hline$\geq 5$ & 54 & $0.57(0.06)$ & 0.032 & 0.064 & 62 & $0.33(0.06)$ & 0.900 & 0.829 \\
\hline
\end{tabular}

${ }^{\star}$ Adjusted for age, race, education, marital status, household income, employment, follow-up time, body mass index, knee injury and knee surgery, smoking, milk and juice intake, total energy intake, baseline Kellgren-Lawrence grade and joint space width (JSW), weight change, the changes of rim distance and beam angle.

and has been associated with increased risk of obesity, type 2 diabetes, cardiovascular disease and poor bone health. $^{10}{ }^{11}{ }^{30}$ Nevertheless, the biological mechanism for soft drinks in the progression of $\mathrm{OA}$ remains unclear. One explanation for the observed findings is that soft drinks may substitute for healthy diet. Soft drink consumption may be associated with decreased intakes of protein, milk and dairy products, fruit juice, fruit and a variety of vitamins and nutrients. $^{12} 31$ One study reported a negative association between soft drink consumption and an overall healthy eating index. ${ }^{32}$ However, in our analysis, the observed effects remained after adjustment for milk and juice intake supports the likelihood that this is not only due to displacement of other healthy beverages in the diet. We considered the extent to which the sugar in soft drinks leads to OA progression. To further evaluate this, we evaluated the relation between fruit juice consumption and OA progression. Fruit juice consumption was not associated with OA progression in our study (results are not shown). It is possible that vitamins, minerals, soluble fibre and phytochemicals in fruit juices may have

\begin{tabular}{|c|c|c|c|c|c|c|}
\hline \multirow[b]{2}{*}{ Soft drinks (times/week) } & \multicolumn{3}{|l|}{ Men } & \multicolumn{3}{|c|}{ Women } \\
\hline & $\mathbf{N}$ & HR (95\% Cl) & $p$ trend & $\mathbf{N}$ & HR (95\% Cl) & $p$ treno \\
\hline \multicolumn{7}{|l|}{ Overall } \\
\hline None & 202 & Referent & & 485 & Referent & \\
\hline$\leq 1$ & 439 & $1.56(1.13$ to 2.16$)$ & & 537 & 0.80 (0.62 to 1.02$)$ & \\
\hline $2-4$ & 140 & 1.55 (1.02 to 2.35$)$ & & 145 & 1.09 (0.77 to 1.54$)$ & \\
\hline$\geq 5$ & 107 & 2.05 (1.32 to 3.19$)$ & 0.002 & 94 & $0.81(0.52$ to 1.26$)$ & 0.539 \\
\hline \multicolumn{7}{|l|}{ Non-obese } \\
\hline None & 113 & Referent & & 279 & Referent & \\
\hline$\leq 1$ & 266 & 1.66 (1.05 to 2.63$)$ & & 277 & 0.71 (0.49 to 1.03 ) & \\
\hline $2-4$ & 85 & 1.45 (0.80 to 2.60$)$ & & 60 & $1.03(0.59$ to 1.80$)$ & \\
\hline$\geq 5$ & 53 & 2.30 (1.22 to 4.34$)$ & 0.025 & 32 & $0.86(0.41$ to 1.84$)$ & 0.559 \\
\hline \multicolumn{7}{|l|}{ Obese } \\
\hline None & 89 & Referent & & 206 & Referent & \\
\hline$\leq 1$ & 173 & 1.58 (0.99 to 2.52$)$ & & 260 & 0.88 (0.63 to 1.25$)$ & \\
\hline $2-4$ & 55 & 1.77 (0.96 to 3.28$)$ & & 85 & $1.17(0.74$ to 1.84$)$ & \\
\hline$\geq 5$ & 54 & 1.90 (1.03 to 3.52$)$ & 0.033 & 62 & $0.87(0.50$ to 1.52$)$ & 0.965 \\
\hline
\end{tabular}


beneficial effects counterbalancing potential adverse effects of sugars.

Previous studies demonstrated that weight gain and obesity may increase risk of joint space loss, suggestive of cartilage loss, as visualised on radiographs, ${ }^{7} 33$ 34 though these findings are not universally reported. Nevertheless, our mediation analysis indicated that the indirect effect through BMI was modest and the association between soft drinks and OA progression remained after adjustment for BMI, weight change and total energy intake suggestive of independent effects of soft drinks. Soft drinks may contain phosphoric acid, which was shown to interfere with calcium absorption and to contribute to imbalances that lead to additional loss of calcium. ${ }^{35}$ It has also been suggested that the high fructose corn syrup used to sweeten carbonated beverages may negatively affect bone. ${ }^{36}$ Long-term effects of soft drinks on OA have not been studied in experimental settings so far, and further research is warranted.

Sex differences have been noted in the prevalence, incidence and severity of OA for many years. ${ }^{37}$ Faber $e t a l^{88}$ found cartilage thickness of the distal femur to be less in women than in men. Other evidences suggested a protective effect of exogenous oestrogen on cartilage and bone turnover. ${ }^{39}$ However, the gender differences in the relationship of soft drink consumption with OA progression are not understood. We found a stronger association between soft drink consumption and JSW change in nonobese men than in obese men. One possible reason is that the effect of soft drink consumption may not be strong enough to provide additional effect beyond obesity.

The strengths of this study include the prospective design, large number of patients with knee OA and the state-of-the-art quantitative measures of structural change from sophisticated image processing technology. The quantitative software-based assessment provides a more precise measure of JSW in millimetres and permits the assessor to document appreciable change in JSW in the tibiofemoral compartment ${ }^{18}{ }^{19}$ In contrast, the semiquantitative approach, for example, the KL grading or the OARSI score, has limitations that lead to insensitivity to changes in status. ${ }^{40}$ The consistent findings from quantitative as well as semiquantitative measures of OA progression increase the reliability of the study. In addition, we excluded knees in which the difference of rim distance between follow-up and baseline visits $\geq 2 \mathrm{~mm}$ and adjusted for changes of rim distance and beam angle in the multivariate models to minimise the possible measurement error of radiographic data.

Owing to the observational nature of the study, patients were not randomly assigned to soft drink groups. We cannot prove that the observed associations are causal because residual confounding could theoretically affect the observed associations. We controlled for potential confounding by most known risk factors that are plausibly associated with soft drink consumption and changes in these variables over time. However, adjustment for baseline covariates may not completely remove the confounding influence. For example, effect of BMI may be lagged, and the cumulative exposure to overweight/obesity may not be perfectly correlated with baseline BMI. Imprecise dietary measurement could potentially have influenced our observed associations. However, random errors in dietary assessment measures might have accounted for a lack of association but not the reverse. Regarding physical activity, PASE can potentially capture all types of activities and allow grading by intensity for elderly. However, questionnaires have obvious weaknesses considering recall and reporting bias. Also PASE may not be sufficient for assessing PA levels and intensity in younger patients with OA. ${ }^{41}$

In conclusion, our study suggested that frequent consumption of sweetened soft drinks may be associated with increased OA progression in men. Replication of these novel findings in other prospective studies demonstrating the reduction in soft drink consumption leads to delay in OA progression are needed to test this hypothesis.

Contributors BL, CBE, JD, KLL and TM conceived the idea of the study and were responsible for the design of the study. BL, OA and F-FZ were responsible for undertaking the data analysis and produced the tables and graphs. The initial draft of the manuscript was prepared by BL, OA and CBE and then circulated repeatedly among all authors for critical revision and all coauthors contributed to the interpretation of the results, read and approved the final manuscript.

Funding This study was supported by National Heart, Lung and Blood Institute (Contract number: HHSN268201000020C, Reference Number: BAA-NHLBI-AR1006). The OAI is a public-private partnership comprised of five contracts (N01-AR-2-2258; N01-AR-2-2259; N01-AR-2-2260;

N01-AR-2-2261; N01-AR-2-2262) funded by the National Institutes of Health, a branch of the Department of Health and Human Services and conducted by the OAI Study Investigators. Private funding partners include Pfizer, Inc; Novartis Pharmaceuticals Corporation; Merck Research Laboratories and GlaxoSmithKline. Private sector funding for the OAI is managed by the Foundation for the National Institutes of Health.

Competing interests None.

Ethics approval OAI was approved by the Institutional Review Board, the University of California, San Francisco (UCSF) and its affiliates. UCSF holds Office of Human Research Protections Federal wide assurance number FWA00000068.

Provenance and peer review Not commissioned; externally peer reviewed.

Data sharing statement No additional data are available.

Open Access This is an Open Access article distributed in accordance with the Creative Commons Attribution Non Commercial (CC BY-NC 3.0) license, which permits others to distribute, remix, adapt, build upon this work noncommercially, and license their derivative works on different terms, provided the original work is properly cited and the use is non-commercial. See: http:// creativecommons.org/licenses/by-nc/3.0/

\section{REFERENCES}

1. Dunlop DD, Manheim LM, Yelin EH, et al. The costs of arthritis. Arthritis Rheum 2003;49:101-13.

2. Lawrence RC, Felson DT, Helmick CG, et al. Estimates of the prevalence of arthritis and other rheumatic conditions in the United States. Part II. Arthritis Rheum 2008;58:26-35.

3. Reginster JY. The prevalence and burden of arthritis. Rheumatology (Oxford) 2002;41(Supp 1):3-6.

4. Felson DT. An update on the pathogenesis and epidemiology of osteoarthritis. Radiol Clin North Am 2004;42:1-9, v.

5. Garstang SV, Stitik TP. Osteoarthritis: epidemiology, risk factors, and pathophysiology. Am J Phys Med Rehabil 2006;85(Suppl 11):S2-11; quiz S12-14. 
6. Zhang Y, Niu J, Felson DT, et al. Methodologic challenges in studying risk factors for progression of knee osteoarthritis. Arthritis Care Res (Hoboken) 2010;62:1527-32.

7. Felson DT, Goggins J, Niu J, et al. The effect of body weight on progression of knee osteoarthritis is dependent on alignment. Arthritis Rheum 2004;50:3904-9.

8. Nielsen SJ, Popkin BM. Changes in beverage intake between 1977 and 2001. Am J Prev Med 2004;27:205-10.

9. Malik VS, Popkin BM, Bray GA, et al. Sugar-sweetened beverages, obesity, type 2 diabetes mellitus, and cardiovascular disease risk. Circulation 2010;121:1356-64.

10. Malik VS, Popkin BM, Bray GA, et al. Sugar-sweetened beverages and risk of metabolic syndrome and type 2 diabetes: a meta-analysis. Diabetes Care 2010;33:2477-83.

11. Wyshak G. Teenaged girls, carbonated beverage consumption, and bone fractures. Arch Pediatr Adolesc Med 2000;154:610-13.

12. Vartanian LR, Schwartz MB, Brownell KD. Effects of soft drink consumption on nutrition and health: a systematic review and meta-analysis. Am J Public Health 2007;97:667-75.

13. McAlindon TE, Felson DT, Zhang $Y$, et al. Relation of dietary intake and serum levels of vitamin $D$ to progression of osteoarthritis of the knee among participants in the Framingham Study. Ann Intern Med 1996;125:353-9.

14. McAlindon TE, Jacques $P$, Zhang $Y$, et al. Do antioxidant micronutrients protect against the development and progression of knee osteoarthritis? Arthritis Rheum 1996;39:648-56.

15. Simard JF, Karlson EW, Costenbader $\mathrm{KH}$, et al. Perinatal factors and adult-onset lupus. Arthritis Rheum 2008;59:1155-61.

16. The osteoarthritis initiative protocol for the cohort study. http://oai. epi-.csf.org/datarelease/docs/StudyDesignProtocol.pdf (accessed 25 Apr 2012)

17. Guermazi A, Hunter DJ, Roemer FW. Plain radiography and magnetic resonance imaging diagnostics in osteoarthritis: validated staging and scoring. J Bone Joint Surg Am 2009;91(Suppl 1):54-62.

18. Duryea J, Zaim S, Genant HK. New radiographic-based surrogate outcome measures for osteoarthritis of the knee. Osteoarthritis Cartilage 2003;11:102-10

19. Sharp JT, Angwin J, Boers M, et al. Computer based methods for measurement of joint space width: update of an ongoing OMERACT project. J Rheumatol 2007;34:874-83.

20. Duryea J, Neumann G, Niu J, et al. Comparison of radiographic joint space width with magnetic resonance imaging cartilage morphometry: analysis of longitudinal data from the Osteoarthritis Initiative. Arthritis Care Res (Hoboken) 2010;62:932-7.

21. Block G, Hartman AM, Naughton D. A reduced dietary questionnaire: development and validation. Epidemiology 1990;1:58-64.

22. Block G, Hartman AM, Dresser CM, et al. A data-based approach to diet questionnaire design and testing. Am J Epidemiol 1986;124:453-69.

23. Washburn RA, Smith KW, Jette AM, et al. The Physical Activity Scale for the Elderly (PASE): development and evaluation. J Clin Epidemiol 1993;46:153-62.
24. Johansen KL, Painter P, Kent-Braun JA, et al. Validation of questionnaires to estimate physical activity and functioning in end-stage renal disease. Kidney Int 2001;59:1121-7.

25. Issa SN, Sharma L. Epidemiology of osteoarthritis: an update. Curr Rheumatol Rep 2006;8:7-15

26. Baron RM, Kenny DA. The moderator-mediator variable distinction in social psychological research: conceptual, strategic, and statistical considerations. J Pers Soc Psychol 1986;51:1173-82.

27. Lin DY. Cox regression analysis of multivariate failure time data: the marginal approach. Stat Med 1994;13:2233-47.

28. Yusuf E, Kortekaas MC, Watt I, et al. Do knee abnormalities visualised on $\mathrm{MRI}$ explain knee pain in knee osteoarthritis? A systematic review. Ann Rheum Dis 2011;70:60-7.

29. Driban JB, Lo GH, Lee JY, et al. Quantitative bone marrow lesion size in osteoarthritic knees correlates with cartilage damage and predicts longitudinal cartilage loss. BMC Musculoskelet Disord 2011;12:217.

30. Tucker KL. Dietary intake and bone status with aging. Curr Pharm Des 2003;9:2687-704.

31. Marshall TA, Eichenberger Gilmore JM, Broffitt B, et al. Diet quality in young children is influenced by beverage consumption. J Am Coll Nutr 2005;24:65-75.

32. Rodriguez-Artalejo F, Garcia EL, Gorgojo L, et al. Consumption of bakery products, sweetened soft drinks and yoghurt among children aged 6-7 years: association with nutrient intake and overall diet quality. Br J Nutr 2003;89:419-29.

33. Wolfe F, Lane NE. The long term outcome of osteoarthritis: rates and predictors of joint space narrowing in symptomatic patients with knee osteoarthritis. J Rheumatol 2002;29:139-46.

34. Dougados M, Gueguen A, Nguyen M, et al. Longitudinal radiologic evaluation of osteoarthritis of the knee. J Rheumatol 1992;19:378-84.

35. Amato D, Maravilla A, Montoya C, et al. Acute effects of soft drink intake on calcium and phosphate metabolism in immature and adult rats. Rev Invest Clin 1998;50:185-9.

36. Milne DB, Nielsen FH. The interaction between dietary fructose and magnesium adversely affects macromineral homeostasis in men. J Am Coll Nutr 2000;19:31-7.

37. Srikanth VK, Fryer JL, Zhai G, et al. A meta-analysis of sex differences prevalence, incidence and severity of osteoarthritis. Osteoarthritis Cartilage 2005;13:769-81.

38. Faber SC, Eckstein F, Lukasz S, et al. Gender differences in knee joint cartilage thickness, volume and articular surface areas: assessment with quantitative three-dimensional MR imaging. Skeletal Radiol 2001;30:144-50.

39. Linn S, Murtaugh B, Casey E. Role of sex hormones in the development of osteoarthritis. Pm R 2012;4(Suppl 5):S169-173.

40. Altman RD, Gold GE. Atlas of individual radiographic features in osteoarthritis, revised. Osteoarthritis Cartilage 2007;15(Suppl A):A1-56.

41. Ida Svege I, Kolle E, Risberg MA. Reliability and validity of the Physical Activity Scale for the Elderly (PASE) in patients with hip osteoarthritis. BMC Musculoskelet Disord 2012;13:26. 